ANNALES

POLONICI MATHEMATICI

XLV (1985)

\title{
Riemann domains over Stein spaces
}

\author{
by Nguyen VAN Khue (Warszawa)
}

\begin{abstract}
It is proved that a complex manifold $X$ having an envelope of holomorphy is Stein if and only if $\operatorname{dim} H^{q}\left(X, \mathcal{O}_{x}\right)<\infty$ for $q=1, \ldots, \operatorname{dim}_{C} X-1$.

Let $\tilde{X}$ and $X$ be analytic spaces. We say that $\tilde{X}$ is a Riemann domain over $X$ if there exists a local isomorphism $\pi: \hat{X} \rightarrow X$. In this note the two following theorems are proved.

Theorem A. Let $\tilde{X}$ be Riemann domain over a Stein space $X$ Let

$$
H^{q}\left(\tilde{X}, \mathcal{O}_{X}\right)=0 \quad \text { for } q=1, \ldots, \operatorname{dim}_{c} \tilde{X}-1 .
$$
\end{abstract}

Then

(i) $\mathcal{O}(\tilde{X})$ separates points,

(ii) for every continuous character $\varphi$ on $\dot{\mathcal{C}}(\hat{X})$ there exists $a z \in \hat{X}$ such that $\varphi(\sigma)=\sigma(z)$ for all $\sigma \in \mathcal{O}(\bar{X})$.

Theorem B. Let $\tilde{X}$ be a Riemann domain over a Stein manifold $X$. Then the following conditions are equivalent:

(i) $\tilde{X}$ is Stein,

(ii) for every discrete sequence $\left\{z_{n}\right\}$ in $\hat{X}$ there exists a holomorphic function $f$ on $\bar{X}$ such that $f\left(z_{1}\right) \neq 0$ and $f\left(z_{n}\right)=0$ for $n \geqslant 2$,

(iii) for every coherent analytic sheaf $\mathscr{S}$ over $\bar{X}$ and for every Stein open subset $G$ of $\tilde{X}$ the canonical map

$$
H^{0}(\tilde{X}, \mathscr{S}) \otimes_{\mathcal{O}(\bar{X})} \mathcal{O}(G) \rightarrow H^{0}(G, \mathscr{S})
$$

has dense image.

1. The tensor product of Fréchet modules. In this section we recall the definition and some properties of the tensor product of Fréchet modules. The details about this one can find in [4]. All algebras are assumed to be commutative with unit element. Let $B$ be a Fréchet algebra. By $\mathscr{C}(B)$ we denote the category of Fréchet algebras and continuous $B$-linear maps. A complex

$$
\ldots \rightarrow M_{n+1} \stackrel{d_{n+1}}{\longrightarrow} M_{n} \stackrel{d_{n}}{\rightarrow} M_{n-1} \rightarrow \ldots
$$


in $\mathscr{C}(B)$ is called direct if for each $n$ there exists a continuous $C$-linear map $h_{n}: M_{n} \rightarrow M_{n+1}$ such that

$$
d_{n+1} h_{n}+h_{n-1} d_{n}=\mathrm{id} .
$$

A Fréchet $B$-module $P$ is said to be free if it is isomorphic to $B \hat{\otimes}_{\pi} A$ where $A$ is a Fréchet space and $B \hat{\otimes}_{\pi} A$ denotes the projective tensor product of $B$ and $A$. We say that $P$ is a $D$-projective Fréchet $B$-module if for every direct sequence $M \stackrel{g}{\rightarrow} M^{\prime \prime} \rightarrow 0$ in $\mathscr{C}(B)$ the map $\dot{g}: \operatorname{HOM}_{B}(P, M) \rightarrow \operatorname{HOM}_{B}\left(P, M^{\prime \prime}\right)$ induced by $g$ is surjective, where $\operatorname{HOM}_{B}(P, M)$ denotes the $B$-module of continuous $B$-linear maps from $P$ into $M$. Note that every free Fréchet $B$ module is $D$-projective.

Let $E \in \mathscr{C}(B)$. A complex

$$
P(E): \ldots \rightarrow P_{n} \stackrel{d_{n}}{\rightarrow} \ldots \rightarrow P_{1} \stackrel{d_{1}}{\rightarrow} P_{0} \rightarrow 0
$$

in $\mathscr{C}(B)$ is called a $D$-projective resolution of $E$ if

(i) $\boldsymbol{P}_{j}$ is $D$-projective for $j \geqslant 1$,

(ii) there exists a continuous $B$-linear map $\mathscr{E}: P_{0} \rightarrow E$ such that the complex $P(E) \stackrel{\mathscr{S}}{\rightarrow} E \rightarrow 0$ is exact. Moreover, if $P(E) \stackrel{\&}{\rightarrow} E \rightarrow 0$ is direct, then $P(E)$ is called a direct $D$-projective resolution of $E$. Setting

$$
\begin{aligned}
& d_{n}\left(a_{0} \otimes \ldots \otimes a_{n} \otimes u\right) \\
& =\sum_{j=1}^{n-1}(-1)^{j-1} a_{0} \otimes \ldots \otimes a_{j-1} a_{j} \otimes \ldots \otimes a_{n} \otimes u(-1)^{n} a_{0} \otimes \ldots \otimes a_{n} u, \\
& h_{n}(u)=1 \otimes u, \quad \mathscr{E}(a \otimes u)=a u
\end{aligned}
$$

we get a direct free resolution of $E$

$$
F(E): \ldots \rightarrow B \dot{\otimes}_{\pi} B \dot{\otimes}_{\pi} E \rightarrow B \hat{\otimes}_{\pi} E \rightarrow E \rightarrow 0,
$$

$F(E)$ is said to be the canonical free resolution of $E$. Hence for the covariant functor

$$
E \otimes_{B}: \mathscr{f}(B) \rightarrow \mathscr{H}(B): M \mapsto E \dot{\otimes}_{B} M=E \dot{\otimes}_{\pi} M / \mathrm{Im} d
$$

where

$$
d: E \hat{\bigotimes}_{\pi} B \hat{\otimes}_{\pi} M \rightarrow E \hat{\otimes}_{\pi} M, \quad d(u \otimes a \otimes m)=a u \otimes m-u \otimes a m,
$$

and $\mathscr{M}(B)$ is the category of $B$-modules we can construct the left derived functors

$$
\operatorname{Tôr}_{q}^{B}(E,): \mathscr{G}(B) \rightarrow \mathscr{H}(B)
$$


of $E \hat{\otimes}_{B}$ putting

$$
\text { Tôr } r_{q}^{B}(E, M)=H_{q}\left(F(E) \hat{\otimes}_{B} M\right) \text {. }
$$

It is known [4] that

1.1. Proposition.

(i) $\operatorname{Tô}_{0}^{B}(E)=,E \hat{\otimes}_{B}$,

(ii) $\operatorname{Tôr}_{q}^{B}(E, F)=\operatorname{Tor}_{q}^{B}(F, E)$ for $q \geqslant 0$ and $E, F \in \mathscr{C}(B)$,

(iii) for every direct sequence

$$
0 \rightarrow M^{\prime} \rightarrow M \rightarrow M^{\prime \prime} \rightarrow 0
$$

in $\mathscr{C}(B)$ there exists an exact homology sequence

$$
\ldots \rightarrow \mathrm{Tôr}_{1}^{B}\left(E, M^{\prime \prime}\right) \rightarrow E \hat{\otimes}_{B} M^{\prime} \rightarrow E \hat{\otimes}_{B} M \rightarrow E \hat{\otimes}_{B} M^{\prime \prime} \rightarrow 0,
$$
then

(v) if $P(E)$ is a nuclear $D$-projective resolution of $E$, i.e., $P_{j}$ are nuclear,

$$
\text { Tôr }{ }_{g}^{B}(E, M)=H_{4}\left(P(E) \otimes_{B} M\right) \text {. }
$$

2. Sheaves $F \otimes_{\mathcal{O}(X)} \mathcal{O}_{X}$. Let $X$ be an analytic space having a countable topology and let $\mathcal{O}_{X}$ (shortly $\left({ }^{(}\right)$denote the structure sheaf of $X$. Let $F$ be a Fréchet $B$-module. By $F \hat{\otimes}_{a(X)} \mathcal{O}$ we denote the sheaf over $X$ given by the formula

$$
U \mapsto F \hat{\otimes}_{\mathcal{O}(x)} \mathcal{O}(U) .
$$

2.1. Proposition. Let $\mathscr{S}$ be an analytic Fréchet sheaf over $X$. Let $\mathscr{S}$ satisfy the following condition:

For every relatively compact Stein open set $U \subset X$ there exists a Fréchet $\mathcal{O}(U)$-module $F(U)$ such that

$$
\mathscr{S} \mid U=F(U) \hat{\otimes}_{\mathcal{Q} U)} \mathcal{O} \quad \text { and } \quad \operatorname{Tôr}_{1}^{\mathcal{Q}(U)}(F(U), \mathcal{O}(V))=0
$$

for all Stein open sets $V \subset U$.

Then for every Stein open set $G$ in $X$ there exists a Fréchet $\mathcal{O}(G)$-module $F(G)$ such that

$$
S \mid G \cong F(G) \hat{\otimes}_{\mathcal{O}(G)} \mathcal{O} \quad \text { and } \quad \operatorname{Tô}_{1}^{\mathcal{O}(G)}(F(G), \mathcal{O}(V))=0
$$

for all relatively compact Stein open set $V \subset G$.

We need the following

2.2. Lemma [4] (Mittag-Leffler). Let $0 \rightarrow\left\{E_{j}^{\prime}\right\} \rightarrow\left\{E_{j}\right\} \rightarrow\left\{E_{j}^{\prime \prime}\right\} \rightarrow 0$ be an exact sequence of projective systems of Fréchet spaces such that for all $i, j, i \leqslant j$, the map $E_{j}^{\prime} \rightarrow E_{i}^{\prime}$ has dense image. Then the sequence

is exact.

$$
0 \rightarrow \underset{\leftarrow}{\lim } E_{j}^{\prime} \rightarrow \lim _{\leftarrow} E_{j} \rightarrow \lim _{\leftarrow} E_{j}^{\prime \prime} \rightarrow 0
$$


From the definition of the projective tensor product we get the following

2.3. LemMA. Let $\left\{E_{j}\right\}$ and $\left\{F_{j}\right\}$ be projective systems of Fréchet spaces such that for all $i \leqslant j$ the maps $E_{j} \rightarrow E_{i}$ and $F_{j} \rightarrow F_{i}$ have dense images. Then

$$
\lim _{\leftarrow}\left(E_{j} \hat{\otimes}_{\pi} F_{j}\right)=\left(\lim _{\leftarrow} E_{j}\right) \hat{\otimes}_{\pi}\left(\lim _{\leftarrow} F_{j}\right) \text {. }
$$

Pro of of Proposition 2.1. (a) First we show that for every relatively compact Stein open set $U \subset X$ and for every Stein open set $V \subset U$ we have

$$
H^{0}(V, \mathscr{S})=H^{0}(U, \mathscr{S}) \hat{\otimes}_{\mathcal{O U}} \mathcal{O}(V) \text {. }
$$

Obviously (2.3) holds in the case where $F(U)$ is free. In general, consider the direct sequences.

$$
0 \rightarrow F_{i} \rightarrow P_{i-1} \rightarrow F_{i-1} \rightarrow 0,
$$

$i=1,2$, where $F_{0}=F(U) ; P_{0}$ and $P_{1}$ are free Fréchet $\mathcal{O}(U)$-modules. By (2.1) and by the exactness of sequences (1.2) associated with the direct sequences $(2.4)$ we get the exact sequences

$$
0 \rightarrow F_{i} \hat{\otimes}_{\mathcal{O U}} \mathcal{O} \rightarrow P_{i-1} \hat{\otimes}_{\mathcal{O}(U)} \mathcal{O} \rightarrow F_{i-1} \hat{\otimes}_{\mathcal{O U}} \mathcal{O} \rightarrow 0 \text {. }
$$

Since $H^{1}\left(U, F_{i} \hat{\otimes}_{\mathcal{O} U)} \mathcal{O}\right)=0$ (Proposition 3.1) we get the commutative and exact diagram

$$
\begin{aligned}
& F_{i} \hat{\otimes}_{Q(U)} \mathcal{O}(V) \longrightarrow P_{i-1} \hat{\otimes}_{Q U)} \mathcal{O}(V) \longrightarrow F_{i-1} \hat{\otimes}_{\mathcal{Q}(U)} \mathcal{O}(V) \rightarrow 0
\end{aligned}
$$

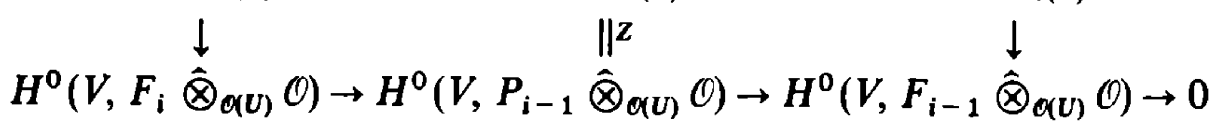

Therefore it follows that $H^{\circ}(V, \mathscr{S})=F_{0} \hat{\otimes}_{\mathcal{O}(U)} \mathcal{O}(V)$.

(b) We write $G=\bigcup_{n=1}^{\infty} G_{n}$ where $V \subset G_{1}$ and $\left\{G_{n}\right\}$ is an increasing sequence of relatively compact holomorphically convex domains in $G$. For each $n$ consider the canonical free resolutions of the Fréchet $\mathcal{O}\left(G_{n}\right)$ and $\mathcal{O}(G)$-modules $\mathcal{O}(V)$

$$
\begin{aligned}
\ldots & \stackrel{d_{1}}{\rightarrow} \mathcal{O}(G) \hat{\otimes}_{\pi} \mathcal{O}(V) \rightarrow \mathcal{O}(V) \rightarrow 0 \\
\ldots \stackrel{d_{1}^{n}}{\rightarrow} \mathcal{O}\left(G_{n}\right) \hat{\otimes}_{\pi} \mathcal{O}(V) & \rightarrow \mathcal{O}(V) \rightarrow 0
\end{aligned}
$$

By (a), Tôr ${ }_{1}^{\left.Q G_{n}\right)}\left(H^{0}\left(G_{n}, \mathscr{S}\right) \mathcal{O}(V)\right)=0$ and the restriction maps $H^{0}\left(G_{n+1}, \mathscr{S}\right)$ $\rightarrow H^{0}\left(G_{n}, \mathscr{S}\right)$ have dense images. Hence, using Lemmas 2.2 and 2.3 to the exact sequence of projective systems

$$
\begin{aligned}
\left\{\mathcal{O}\left(G_{n}\right)^{\otimes 3} \hat{\otimes}_{n} \mathscr{S}\left(G_{n}\right)\right\} \rightarrow\left\{\mathcal{O}\left(G_{n}\right)^{\otimes 2} \hat{\otimes}_{n} \mathscr{S}\left(G_{n}\right)\right\} & \rightarrow\left\{\mathcal{O}\left(G_{n}\right) \hat{\otimes}_{n} \mathscr{S}\left(G_{n}\right)\right\} \rightarrow\{\mathscr{S}(V)\} \rightarrow 0,
\end{aligned}
$$

where $\mathcal{O}\left(G_{n}\right)^{\otimes i}=\mathcal{O}\left(G_{n}\right) \underbrace{\hat{\otimes}_{\pi} \ldots \hat{\otimes}_{\pi}}_{i} \mathcal{O}\left(G_{n}\right)$, we get $(2.2)$. 
The proposition is proved.

2.4. Proposition. For every coherent analytic sheaf $\mathscr{S}^{\prime \prime}$ over a Stein space $X$ there exists an exact sequence

$$
0 \rightarrow \mathscr{S}^{\prime} \rightarrow \mathscr{S} \rightarrow \mathscr{S}^{\prime \prime} \rightarrow 0
$$

of analytic Fréchet sheaves over $X$ such that $\mathscr{S}^{\prime}, \mathscr{S}$ and $\mathscr{S}^{\prime \prime}$ satisfy condition (2.2) and $\mathscr{S}=\mathcal{O} \in l_{1}$.

Proof. (a) First observe that for every coherent analytic sheaf $\mathscr{S}$ over $X$ and for every Fréchet space $F$ the analytic Fréchet sheaf $\mathscr{S} \in F$ over $X$ given by the formula

$$
U \mapsto H^{0}(U, \mathscr{S}) \hat{\otimes}_{\pi} F
$$

satisfies condition (2.1) and hence by Proposition 2.1 we infer that $\mathscr{S} \in F$ satisfies (2.2).

(b) We write $X=\bigcup_{j=1}^{\infty} G_{j}$, where $\left\{G_{j}\right\}$ is an increasing sequence of relatively compact open sets in $X$. By the Cartan Theorem $\mathrm{A}$ it follows that there exists a sequence $\left\{\sigma_{j}\right\} \subset H^{0}\left(X, \mathscr{S}^{\prime \prime}\right)$ such that $\left\{\sigma_{j z}\right\}$ generates $\mathscr{S}_{z}^{\prime \prime}$ for $z \in X$. Hence the formula

$$
g_{U}\left(\left\{\alpha_{j}\right\}\right)=\sum_{j=1}^{\infty} \alpha_{j} \sigma_{j} \mid U / l \rightarrow\left\|\sigma_{j}\right\|_{G_{j}}
$$

defines a morphism $g$ from $\mathscr{S}=\mathscr{O} \in l_{1}$ onto $\mathscr{S}^{\prime \prime}$. It is easy to see that for every relatively compact Stein open set $U \subset X$ the sequence

$$
0 \rightarrow H^{0}\left(U, \mathscr{S}^{\prime}\right) \rightarrow H^{0}(U, \mathscr{S}) \rightarrow H^{0}\left(U, \mathscr{S}^{\prime \prime}\right) \rightarrow 0
$$

ist exact. Hence by the exactness of the sequence (1.2) associated with the exact sequence (2.7) and by (a) we get

$$
\mathscr{S}^{\prime} \mid U \cong H^{0}\left(U, \mathscr{S}^{\prime}\right) \hat{\otimes}_{\mathcal{G}(U)} \mathcal{O} \quad \text { and } \quad \operatorname{Tôr}_{1}^{O(U)}\left(H^{0}\left(U, \mathscr{S}^{\prime}\right), \mathcal{O}(V)\right)=0
$$

for every Stein open set $V \subset U$. Hence, by Proposition 2.1 it follows that $\mathscr{S}^{\prime}$ satisfies condition (2.2).

The proposition is proved.

\section{Cohomology groups of sheaves $F \hat{\otimes}_{\mathcal{O}(X)} \mathcal{O}$.}

3.1. Proposition. Let $X$ be an analytic space having a countable topology and let $F$ be a Fréchet $\mathcal{O}(X)$-module such that $\operatorname{Tô}_{1}^{\mathcal{O}(X)}(F, \mathcal{O}(V))=0$ for every sufficiently small Stein open set $V \subset X$. Let $\tilde{X}$ be Riemann domain over $X$ such that $H^{q}\left(\tilde{X}, \mathcal{O}_{\bar{X}}\right)=0$ for $q \geqslant 1$. Then

$$
H^{q}\left(\tilde{X}, F \hat{\otimes}_{\mathcal{O}(X)} \mathcal{O}_{\mathfrak{X}}\right)=0 \quad \text { for } q \geqslant 1 \text {. }
$$


Proof. (a) First we assume that $F=\mathcal{O}(X) \hat{\otimes}_{\pi} A$ where $A$ is a Fróchet space. Then $F \hat{\otimes}_{\mathcal{O}(X)} \mathcal{O}_{\tilde{X}}=\mathcal{O}_{\tilde{X}} \in A$. Let $\mathscr{U}$ be a Stein open covering of $\tilde{X}$. Then $\mathcal{U}$ is a Leray covering for $\mathcal{O}_{\mathbb{X}}$ and hence by hypothesis the sequence

$$
0 \rightarrow \mathcal{O}(\tilde{X}) \rightarrow C^{0}\left(U, \mathcal{O}_{\tilde{X}}\right) \rightarrow \ldots
$$

is exact. Hence the sequence

$$
0 \rightarrow \mathcal{O}(\tilde{X}) \hat{\otimes}_{\pi} A \rightarrow C^{0}\left(U, \mathcal{O}_{\tilde{X}} \in A\right) \rightarrow \ldots
$$

is also exact. Since $\mathscr{U}$ is a Leray covering for $\mathcal{O}_{\mathbb{X}} \in A,[1]$, it follows that $H^{q}\left(\tilde{X}, F \hat{\otimes}_{\mathcal{O}(X)} \mathcal{O}_{\tilde{X}}\right)=0$ for $q \geqslant 1$.

(b) In general, consider the direct sequences (2.1) where $i=1,2, \ldots$ By hypothesis the sequences (2.5) are exact. Hence by (a) we have

$$
H^{q}\left(\tilde{X}, F \hat{\otimes}_{\mathcal{O}(X)} \mathcal{O}_{\tilde{X}}\right) \cong \ldots \cong H^{q+p}\left(\tilde{X}, F_{p} \hat{\otimes}_{\mathcal{O}(X)} \mathcal{O}_{\tilde{X}}\right)=0
$$

for sufficiently large $p$ where $q \geqslant 1$. The proposition is proved.

4. Proof of Theorem A. By a theorem of Siu we have $H^{q}\left(\tilde{X}, \mathcal{O}_{\tilde{X}}\right)=0$ for $q \geqslant \operatorname{dim}_{c} \tilde{X},[7]$.

(i) We prove (i) by induction on $\operatorname{dim} \tilde{X}$. The case $\operatorname{dim} \tilde{X}=0$ is trivial. Assume that (i) has been established for all $\tilde{X}$ with $\operatorname{dim} \tilde{X}<n$ and let $(\tilde{X}, \pi, X)$ be a Riemann domain as in Theorem $\mathrm{A}$ with $\operatorname{dim} \tilde{X} \leqslant n$.

(a) First we show that if $\tilde{Z}$ be a Riemann domain over a Stein space $Z$ and if $H^{q}\left(\tilde{Z}, \mathcal{O}_{Z}\right)=0$ for $q \geqslant 1$, then $H^{q}\left(\tilde{Z}_{\text {red }}, \mathcal{O}_{Z_{\text {red }}}\right)=0$ for $q \geqslant 1$. Let $\tilde{N}$ (resp. $N$ ) denotes the nilradical of $\mathcal{O}_{\tilde{Z}}$ (resp. that of $\mathcal{O}_{Z}$ ). By Proposition 2.1 we have $N \cong H^{0}(Z, N) \hat{\otimes}_{m(Z)} \mathcal{O}_{z}$ and hence $\tilde{N} \cong H^{0}(Z, N) \hat{\otimes}_{a(Z)} \mathcal{O}_{z}$. Thus by Propositions 2.1 and 3.1 we get $H^{q}(\tilde{Z}, \tilde{N})=0$ for $q \geqslant 1$. Then by the exactness of the cohomology sequence associated with the exact sequence

$$
0 \rightarrow \tilde{N} \rightarrow \mathcal{O}_{\tilde{Z}} \rightarrow \mathcal{O}_{\tilde{z}_{\text {red }}} \rightarrow 0
$$

it follows that $H^{q}\left(\tilde{Z}_{\text {red }}^{+}, \mathcal{O}_{Z_{\text {red }}}\right)=0$ for $q \geqslant 1$ and the restriction map $\mathcal{O}(\tilde{Z})$ $\rightarrow \mathcal{O}\left(\tilde{Z}_{\text {red }}\right)$ is surjective. Thus we can assume that $\tilde{X}$ is reduced.

(b) Choose a holomorphic function $g$ on $X$ which is not constant on every irreducible component of $X$. Since $\tilde{X}$ is reduced the sequence

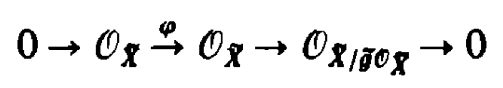

is exact, where $\tilde{g}=g \pi$, and $\varphi$ is defined by multiplication by $\tilde{g}$. By hypothesis we infer that $H^{q}\left(V(\tilde{g}), \mathcal{C}_{\mathbb{R} / g^{g} \mathfrak{q}}\right)=0$ for $q \geqslant 1$, where $V(\tilde{g})=\tilde{g}^{-1}(0)$. By (a) we get $H^{q}\left(V(\tilde{g}), \mathcal{O}_{V(g)}\right)=0$ for $q \geqslant 1$. Since $(V(\tilde{g}), \pi, V(g))$, is a Riemann domain over the Stein space $V(g)$ of dimension $<n$ it follows that $\mathcal{O}(V(\tilde{g}))$ separates points.

(c) We prove that the restriction map $\mathcal{O}(\tilde{X}) \rightarrow \mathcal{O}(V(\tilde{g}))$ is surjective. Let $h \in \mathcal{O}(V(\tilde{g}))$. By the proof in (a) it follows that $H^{1}(V(\tilde{g})$, Ker $\eta)=0$, where $\eta$ 
denotes the canonical map from $\mathcal{O}_{\tilde{X} / \tilde{g} \odot \tilde{X}}$ onto $\mathcal{O}_{V(\bar{\theta})}$. Hence $h=\eta k$ for some $k \in H^{0}\left(V(\tilde{g}), \mathcal{O}_{\mathcal{S}_{/ \tilde{\theta}} \mathcal{X}}\right)$. Take a Stein open covering $\mathscr{U}=\left\{U_{j}\right\}_{j=1}^{\infty}$ of $\tilde{X}$ and $k_{j} \in \mathcal{O}\left(U_{j}\right)$ such that

$$
k_{j}\left|U_{j} \cap V(\tilde{g})=k\right| U_{j} \cap V(\tilde{g})
$$

Then

$$
k_{i}-k_{j}=\tilde{g} g_{i j} \quad \text { on } U_{i} \cap U_{j} \quad \text { for some } g_{i j} \in \mathcal{O}\left(U_{i} \cap U_{j}\right) \text {. }
$$

Since $\tilde{X}$ is reduced and $V(\tilde{g})$ is nowhere dense it follows that $\left(g_{i j}\right) \in Z^{1}\left(\mathscr{U}, \mathcal{O}_{\bar{x}}\right)$. Hence $\left(g_{i j}\right)=\delta\left(g_{i}\right)$ for some $\left(g_{i}\right) \in C^{0}\left(\mathscr{U}, \mathcal{O}_{\tilde{X}}\right)$. If we define

$$
\tilde{h}=k_{i}-\tilde{g} g_{i} \quad \text { on } U_{i}
$$

we get a holomorphic extension of $h$ on $\tilde{X}$.

(d) (i) follows from (b) and (c).

(ii) Let $\varphi$ be a continuous character of $\mathcal{O}(\tilde{X})$. By (a), it is known [3] that $\tilde{X}$ is separated by a finite subset of $\operatorname{Ker} \varphi$. Then, by the above hypothesis and by considering the Koszul complexes of finite subsets of $\operatorname{Ker} \varphi$ separating points of $\tilde{X}$ Nagel proved [5] that (ii) holds for some $z_{0} \in X$.

The theorem is then proved.

5. Proof of Theorem B. (i) $\Rightarrow$ (ii) by Theorem $B$.

(ii) $\Rightarrow$ (i). Since $\tilde{X}$ has an envelope of holomorphy we can assume that $\tilde{X}$ is an open set in a closed submanifold $Z$ of $C^{N}$ for some $N$. Let $\gamma: G \rightarrow Z$ be a holomorphic retraction of an open neighbourhood $G$ of $Z$ in $C^{N}$ onto $Z$ and let $\Omega=\gamma^{-1}(\tilde{X})$. It suffices to show that $\Omega$ is Stein. Assume the contrary, that there exist $p \in \Omega$ and a polydisc $\Delta$ around $p$ with $\Delta \not \Omega$ such that every $f \in \mathcal{O}(\Omega)$ has a Taylor series on $\Delta$ which converges to $F \in \mathcal{O}(\Delta)$. Let $W$ be a component of $\Delta \cap \Omega$ containing $p$. Let $q \in \Delta \backslash \Omega$. Consider the complex line $E$ through $p$ and $q$. On the real segment $p q$ from $p$ to $q$ there exists a first point

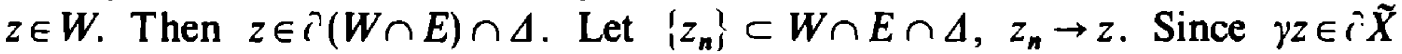
there exists $f \in \mathcal{O}(\Omega)$ such that $f\left(z_{1}^{\prime}\right) \neq 0$ and $f\left(z_{n}^{\prime}\right)=0, n \geqslant 2$ for a subsequence $\left\{z_{n}^{\prime}\right\} \subset\left\{z_{n}\right\}$. Let $F \in \mathcal{O}(\Delta)$ such that $F|W=f| W$. Then $F \mid E \cap W \cap \Delta$ $=0$ and hence $f\left(z_{1}^{\prime}\right)=0$. This contradics the choice of $f$.

(i) $\Rightarrow$ (iii). Consider the exact sequence (2.6) on $\tilde{X}$ for $\mathscr{S}$. By Propositions 2.4 and 3.1 it follows that the restriction map $H^{0}(\tilde{X}, \mathscr{S}) \hat{\otimes}_{\mathcal{O}(\tilde{X})} \mathcal{O}(G)$ $\rightarrow H^{0}(G, \mathscr{S})$ has dense image for every Stein open set $G \subset X$.

(iii) $\Rightarrow$ (ii). Let $\left\{z_{j}\right\}$ be a discrete sequence in $\tilde{X}$. Let $\left\{G_{j}\right\}$ be disjoint Stein open neighbourhoods of $z_{j}$ and let $G=\bigcup_{j=1}^{\infty} G_{j}$. Then $G$ is Stein. Applying (iii) to $G$ and to the coherent analytic sheaf $J$ associated with $\left\{z_{j}\right\}, j \geqslant 2$, we have

$$
g=\lim _{n} \sum_{i} f_{i}^{n} g_{i}^{n}, \quad f_{i}^{n} \in H^{0}(\hat{\bar{X}}, J), g_{i}^{n} \in \mathcal{O}(G)
$$


where $g \in H^{0}(G, J), g\left(z_{1}\right) \neq 0$. It follows that $f_{i_{0}}^{n_{0}}\left(z_{1}\right) \neq 0$ for some $\left(n_{0}, i_{0}\right)$ and hence (ii) holds.

The theorem is proved.

6. An application of Theorem $A$. Let $X$ be a reduced analytic space and $\tilde{X}$ a reduced Stein space of dimension $\operatorname{dim} X$. We say that $\tilde{X}$ be an envelope of holomorphy of $X$ if there exists a holomorphic map $\theta: X \rightarrow \tilde{X}$ with discrete fibre such that the map $\hat{\theta}: \mathcal{O}(\hat{X}) \rightarrow \mathcal{O}(X)$ induced by $\theta$ is surjective. It is known [6] that every Riemann domain over a Stein manifold has an envelope of holomorphy.

Consider the submanifold $X$ of $C^{3} \backslash 0,[6]$, given by

$$
X=\left\{\left(z_{1}, z_{2}, z_{3}\right): z_{3}^{2}=z_{1} z_{2}\right\} \backslash 0 .
$$

Then $\tilde{X}=V\left(z_{3}^{2}-z_{1} z_{2}\right)$ is an envelope of holomorphy of $X$ and, moreover, the restriction map $\mathcal{O}(\bar{X}) \rightarrow \mathcal{O}(X)$ is an isomorphism. This implies that $X$ does not admit an envelope of holomorphy which is a manifold.

In this section we prove the following

6.1. TheOREM. Let $X$ be a complex manifold having an envelope of holomorphy $W$. Then $X$ is Stein if and only if $\operatorname{dim} H^{q}\left(X, \mathcal{O}_{X}\right)<\infty$ for $q=1, \ldots, \operatorname{dim}_{c} X-1$.

Proof. Let $\pi: \tilde{W} \rightarrow W$ be the normalization of $W$. Then $\tilde{W}$ is Stein [2]. Since $\theta: X \rightarrow W$ has discrete fibre, it follows that $\theta^{-1}(N(W))$ is rare in $X$ [2] (Remark 0.43), where $N(W)$ denotes the non-normal locus of $W$. Hence there exists a holomorphic map $\tilde{\theta}: X \rightarrow \tilde{W}$ such that $\pi \tilde{\theta}=\theta$ [2] (Proposition 2.28). Since $\tilde{\theta}$ has discrete fibre, by the normality of $\tilde{W}$ it follows that $\tilde{\theta}$ is open. By Theorem A, Theorem 6.1 is an immediate consequence of the following

6.2. Proposition. Let $Z$ be a complex $K$-separable manifold, i.e., there exists a holomorphic map $\pi=\left(\pi_{1}, \ldots, \pi_{n}\right)$ with discrete fibre from $Z$ into $C^{n}$, and $\operatorname{dim} H^{q}\left(Z, O_{Z}\right)<\infty$ for $q \geqslant 1$. Then

$$
H^{q}\left(Z, \mathcal{O}_{Z}\right)=0 \text { for } q \geqslant 1 \text { and } \mathcal{O}(Z) \text { separates points. }
$$

Proof. Let $g=\left(g_{1}, \ldots, g_{n}\right)$ be a holomorphic map from $C^{n}$ into $C^{n}$ with discrete fibre. Then

$$
\operatorname{dim}_{z} V\left(\tilde{g}_{1}, \ldots, \tilde{g}_{k}\right)=n-k \quad \text { for all } z \in \tilde{Z} .
$$

By (6.1) and by a lemma of Siu [8] it follows that the sequence

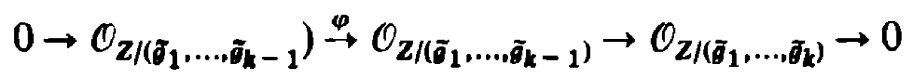

is exact, where $\varphi$ is defined by multiplication by $\tilde{g}_{k}$. Thus, by hypothesis and by induction on $k$ we get

$$
\operatorname{dim} H^{q}\left(Z, \mathcal{O}_{Z /\left(\sigma_{1}, \ldots, \tilde{\sigma}_{k}\right)}\right)<\infty \quad \text { for } q \geqslant 1 \text { and } k=0, \ldots, n .
$$


We prove by the decreasing induction on $k$ the following formulae:

$$
\begin{aligned}
& H^{q}\left(Z, \mathcal{O}_{Z /\left(\mathcal{J}_{1}, \ldots, J_{k}\right)}\right)=0 \quad \text { for } q \geqslant 1, \\
& H^{0}\left(Z, \mathcal{O}_{Z /\left(J_{1}, \ldots, J_{k}\right)}\right) \quad \text { separates points of } V\left(f_{1}, \ldots, f_{k}\right)
\end{aligned}
$$

for all holomorphic maps $f=\left(f_{1}, \ldots, f_{n}\right): C^{n} \rightarrow C^{n}$ with discrete fibre, $(6.3)_{n}$ is trivial. Assume that (6.3) $m$ has been established for $k<m \leqslant n$. Fix $q \geqslant 1$. By (6.2) and since the subspace of $\mathcal{O}\left(C^{n}\right)$ spanned by $\left(f_{k}, f_{k}^{2}, \ldots\right)$ is infinite dimensional we find $f=a_{1} f_{k}+\ldots+a_{p} f_{k}^{p} \in \mathcal{O}\left(C^{\eta}\right) \backslash 0$ such that

$$
f H^{q}\left(Z, \mathcal{O}_{Z /\left(f_{1}, \ldots, f_{k-1}\right)}\right)=0 \text {. }
$$

Since $f_{k}$ is not constant on every irreducible component of $V\left(f_{1}, \ldots, f_{k-1}\right)$ it follows that the map $\left(f_{1}, \ldots, f_{k-1}, f, f_{k+1}, \ldots, f_{n}\right): C^{n} \rightarrow C^{n}$ has discrete fibres. Hence the sequence

$$
0 \rightarrow \mathcal{O}_{z /\left(J_{1}, \ldots, J_{k-1}\right)} \stackrel{\varphi}{\rightarrow} \mathcal{O}_{z /\left(f_{1}, \ldots, f_{k-1}\right)} \rightarrow \mathcal{O}_{z /\left(J_{1}, \ldots, f_{k-1}, \tilde{J}\right)} \rightarrow 0
$$

is exact where $\varphi$ is defined by mutiplication by $f$. By $(6.3)_{k}$ it follows that the map

$$
\hat{\varphi}_{q}: H^{q}\left(Z, \mathcal{O}_{Z /\left(\tilde{J}_{1}, \ldots, J_{k-1}\right)}\right) \rightarrow H^{q}\left(Z, \mathcal{O}_{Z /\left(J_{1}, \ldots, J_{k-1}\right)}\right)
$$

induced by $\varphi$ is surjective. Combining this with the relation $\operatorname{Im} \hat{\varphi}_{q}$ $=\widetilde{f} H^{q}\left(Z, \mathcal{O}_{Z /\left(f_{1}, \ldots, J_{k-1}\right)}\right)=0$ we get $H^{q}\left(Z, \mathcal{O}_{Z /\left(f_{1}, \ldots, f_{k-1}\right)}\right)=0$. This implies also that the restriction map

$$
H^{0}\left(Z, \mathcal{O}_{\left.Z / \mathcal{U}_{1}, \ldots, f_{k-1}\right)}\right) \rightarrow H^{0}\left(Z, \mathcal{O}_{Z /\left(\tilde{f}_{1}, \ldots, f_{k-1}, J^{\prime}\right)}\right)
$$

is surjective. Then by induction hypothesis it is easy to see that $H^{0}\left(Z, \mathcal{O}_{Z /\left(f_{1}, \ldots, f_{k-1}\right)}\right)$ separates points of $V\left(\tilde{f}_{1}, \ldots, \tilde{f}_{k-1}\right)$. Hence $(6.3)_{k-1}$ holds.

The proposition is proved.

6.3. Remark. The proof of Proposition 6.2 is similar to that of the first part of Theorem $B$ in [8].

Since every Riemann domain over a Stein manifold has an envelope of holomorphy the following is an immediate consequence of Theorem 6.1 and Proposition 6.2.

6.4. Corollary [8] (Theorem B). Let $X$ be a Riemann domain over a Stein manifold. Then $X$ is Stein if and only if $\operatorname{dim} H^{q}\left(X, \mathcal{O}_{X}\right)<\infty$ for $q=1, \ldots, \operatorname{dim}_{c} X-1$.

6.5. Proposition. Let $X$ be a normal analytic space of dimension $\leqslant 5$ having an envelope of holomorphy. Then $X$ is Stein if and only if $\operatorname{dim} H^{q}\left(X, O_{X}\right)<\infty$ for $1 \leqslant q<\operatorname{dim}_{C} X$.

Proof. By the proof of Theorem 6.1 there exists an open embedding $\theta$ of $X$ into a normal Stein space $\tilde{W}$ such that the map $\hat{\theta}: \mathcal{O}(\tilde{W} \rightarrow \mathcal{O}(X)$ 
is surjective. Hence by Theorem $\mathrm{A}$ it suffices to check that $H^{q}\left(\bar{X}, \mathcal{O}_{\tilde{X}}\right)=0$ for $q \geqslant 1$ and for every Riemann domain $\bar{X}$ over a Stein space $X$ of dimension $\leqslant 5$ satisfying the condition $\operatorname{dim} H^{q}\left(\bar{X}, \mathcal{O}_{\mathbb{X}}\right)<\infty$ for $1 \leqslant q<\operatorname{dim}_{C} X$. Take a holomorphic function $f$ on $X$ such that $f$ is not constant on every irreducible component of $X$ and $S(X)$ where $S(X)$ denotes the singular locus of $X$. Since the subspace of $\mathcal{O}(X)$ spanned by $\left(f, f^{2}, \ldots\right)$ is infinite dimensional, by hypothesis and by the theorem of Siu [7] there exists $g$ $=\sum_{j=1}^{p} \alpha_{j} f^{j} \neq 0$ such that $\tilde{g} H^{q}\left(\tilde{X}, \mathcal{O}_{\tilde{X}}\right)=0$ for $q \geqslant 1$. Consider the exact sequence

$$
0 \rightarrow \tilde{g} \mathcal{O}_{\mathbf{R}} \rightarrow \mathcal{O}_{\mathbf{R}} \rightarrow \mathcal{O}_{\mathbf{X} / \tilde{\mathrm{g}} \mathrm{R}} \rightarrow 0
$$

where $\varphi$ is denoted by multiplication by $g$. Since $\tilde{g}$ is not constant on every irreducible component of $\vec{X}$ and $S(\tilde{X})$ it follows that supp $\operatorname{Ker} \varphi$ is an analytic subset of $V(\tilde{g}) \cap S(\tilde{X})$ of dimension $\leqslant 3$. Hence, by a theorem of Siu [7] we have $H^{q}(\tilde{X}, \operatorname{Ker} \varphi)=0$ for $q \geqslant 3$. Then, by the exactness of the cohomology sequence it follows that for every $q \geqslant 2$ the map $\hat{\varphi}_{q}: H^{q}\left(\tilde{X}, \mathcal{O}_{\tilde{X}}\right)$ $\rightarrow H^{q}\left(\tilde{X}, g \mathcal{O}_{\tilde{X}}\right)$ induced by $\varphi$ is surjective. By hypothesis, considering the exact sequence

$$
0 \rightarrow \tilde{g} \mathcal{O}_{X} \rightarrow \mathcal{O}_{R} \rightarrow \mathcal{O}_{\boldsymbol{X} / \tilde{\boldsymbol{\theta}} \mathcal{O}_{\mathrm{X}}} \rightarrow 0
$$

we infer that $\operatorname{dim} H^{q}\left(\tilde{X}, \mathcal{O}_{\tilde{X} / \tilde{\theta} \mathcal{O}_{\tilde{R}}}\right)<\infty$ for $q \geqslant 1$. Applying the induction hypothesis to the Riemann domain $\left(V(\tilde{g}), \mathcal{O}_{\boldsymbol{X} / \tilde{g} \mathcal{O}_{\tilde{R}}}\right)$ over the Stein space $\left(V(g), \mathcal{O}_{X / 8 \mathcal{O}_{X}}\right)$ it follows that every map $\hat{i}_{q}: H^{q}\left(\bar{X}, \tilde{g} \mathcal{O}_{\bar{X}}\right) \rightarrow H^{q}\left(\bar{X}, \mathcal{O}_{\bar{X}}\right)$ induced by $i$ is surjective where $q \geqslant 1$. Then, by the inclusion $\operatorname{Im} \hat{i}_{q} \subseteq \tilde{g} H^{q}\left(\tilde{X}, \mathcal{O}_{\tilde{X}}\right)$ we infer that $H^{q}\left(\tilde{X}, \mathcal{O}_{\ell}\right)=0$ for $q \geqslant 1$.

The proposition is proved.

\section{References}

[1] L. Bungart, Holomorphic functions with values in locally convex spaces and applications to integral formules, Trans. Amer. Math. Soc. 111 (1964), 317-343.

[2] G. Fischer, Complex Analytic Geometry, Springer-Verlag, Berlin-Heidelberg-New York 1976.

[3] R. Gunning and H. Rossi, Analytic Functions of Several Complex Variables, Prentice-Hall, Englewood Clifrs, N. J, 1965.

[4] J. Hubar, Transversalite, Seminaire de Geometrie Analytique, Asterique 16 (1974), 33-48.

[5] A. Nagel, Cohomologgy maximal ideals and point evaluations, Proc. Amer. Math. Soc. 42 (1974), 47-50.

[6] H. Rossi, On envelope of holomorphy, Comm. Pure Appl. Math. 16 (1963), 9-19. 
[7] Y. T. Siu, Analytic sheaf cohomology groups of dimension $n$ of $n$-dimensional complex spaces, Trans. Amer. Math. Soc. 143 (1969), 77-94.

[8] -, Non-countable dimension of cohomology groups of analytic sheaves and domained of holomorphy, Math. Z. 102 (1967), 17-29.

INSTITUTE OF MATHEMATICS, POLISH ACADEMY OF SCIENCES

SNIADECKICH 8, 00-950 WARSZAWA

Reçu par la Rédaction le 26.01.1981 